\title{
Effects of signaled free reinforcement on concurrent performances*
}

\section{JULIAN LESLIE and J. R. MILLENSON \\ Oxford University, Oxford, England}

Rats were trained to leverpress concurrently for $4 \%$ and $16 \%$ sucrose solution. This established asymmetric performance. The introduction of a CS paired with free food resulted in an increased preference for the greater reinforcement. This result is discussed in terms of the motivational consequences of Pavlovian conditioning procedures.

A major premise of several behavior theories is the notion that instrumental learning and performance are mediated by classically conditioned central motive or emotional states (Mowrer, 1960; Rescorla \& Solomon, 1967; Bindra \& Palfai, 1967; Hammond, 1970). According to one version of this view, a CS that has been paired with a strong positive or negative reinforcer acquires the ability to arouse a central state that modifies the strengths of a wide range of reinforcers (Millenson, 1967). Because instrumental response probabilities depend upon momentary values of the organism's hierarchy of reinforcers, such a conditioned state temporarily affects the rate of responses maintained by both positive and negative reinforcement. Specifically, it has been suggested that a CS that has been paired with an aversive event enhances the rate of aversively maintained behavior and decreases the rate of behavior maintained by positive reinforcement. Conversely, a CS that has been paired with positive reinforcement might be expected to have the opposite effects (Rescorla \& Solomon, 1967).

There is some empirical support for the first set of predictions. A CS that has preceded shock suppresses responding maintained by positive reinforcement (e.g., Estes \& Skinner, 1941) and can accelerate avoidance responding (Rescorla, 1967), although suppression may occur at high shock intensities (Bryant, 1972; Scobie, 1972). However, the data on the effects of a CS that has preceded positive reinforcement do not, in general, support the hypothesis. While acceleration or facilitation has been found when the CS has been tested in extinction of the baseline operant response (Estes, 1943, 1948), or when the US was qualitatively the same as the reinforcement for the operant response (LoLordo, 1971; Azrin \& Hake, 1969), a number of studies with rats and monkeys have shown that presentation of a short CS paired with positive reinforcement will suppress leverpressing for food on a variable-interval schedule

*Supported in part by grants from the Medical Research Council to Professor $L$. Weiskrantz and from the Science Research Council to J. R. Millenson. We thank Roger Bunce for his valuable assistance.
(Azrin \& Hake, 1969; Meltzer \& Brahlek, 1970; Mizcek \& Grossman, 1971). All these studies used USs that differed from the baseline reinforcer.

Previous studies with $\mathrm{CS}^{+}$-US ${ }^{+1}$ have superimposed it on single operant baselines. However, concurrent reinforcement schedules are not only especially sensitive to changes in reinforcement values (cf. Catania, 1966) but they also afford an opportunity for assessing shifts in preference for one response over the other which might arise from changes in the postulated central emotional states. The present experiment investigated the effects of signaled free reinforcement on an asymmetric iwo-response concurrent reinforcement schedule.

\section{METHOD \\ Subjects}

Three adult male hooded rats, designated R4, R8, and R9, of the strain supplied by Scientific Products Farm, Canterbury, Kent, England were used. They had previously been trained to leverpress for $30 \%$ solution and had had free signaled Noyes pellets. They were maintained at $80 \%$ of their free-feeding body weight throughout the experiment.

\section{Apparatus}

A standard Campden Instruments CI410 two-lever rat chamber with a pellet feeder was used. The chamber was modified by the addition of a brass block under each lever, with a countersunk depression into which $0.1 \mathrm{ml}$ of sucrose solution could be delivered from a solenoid-driven syringe. The CS was produced by a Campden Instruments click generator, running at five clicks per second. This produced a sound level of $80 \mathrm{~dB}$ in the chamber (reference level $=0.002$ dyne $\mathrm{cm}^{-2}$ ).

The reinforcement contingencies and other stimuli in the test chamber were controlled by an on-line PDP-8/S digital computer, programmed in ACT, a problem-oriented language for the control of behavioral experiments (Millenson, 1971). The computer also recorded responses made during the various phases of the experiment.

\section{Procedure}

The rats were retrained to respond concurrently on both levers. In order to establish asymmetric performance, reinforcement for presses on the left lever was by a $4 \%$ sucrose solution (by weight) and reinforcement for presses on the right lever was by a $16 \%$ sucrose solution. Hereafter, the levers are referred to as the $4 \%$ lever and the $16 \%$ lever, respectively. The rats were gradually shifted from continuous reinforcement to a schedule on which each response was reinforced after a variable interval, with a mean of $1 \mathrm{~min}$. Reinforcements were assigned with equal probability to each response by a program similar to that used by Stubbs \& Pliskoff (1969). Once a reinforcement was made available for one response, none were set up for the other response until that reinforcement had been delivered. This procedure fixed the relative rates of reinforcement for the two responses at the values determined by the program, regardless of the animal's rates of responding. To strengthen schedule control (cf. Shull \& Pliskoff, 1967), there was a change-over delay (COD) of $1 \mathrm{sec}$, following a switch from one lever to the other, during which reinforcement could never be obtained. 


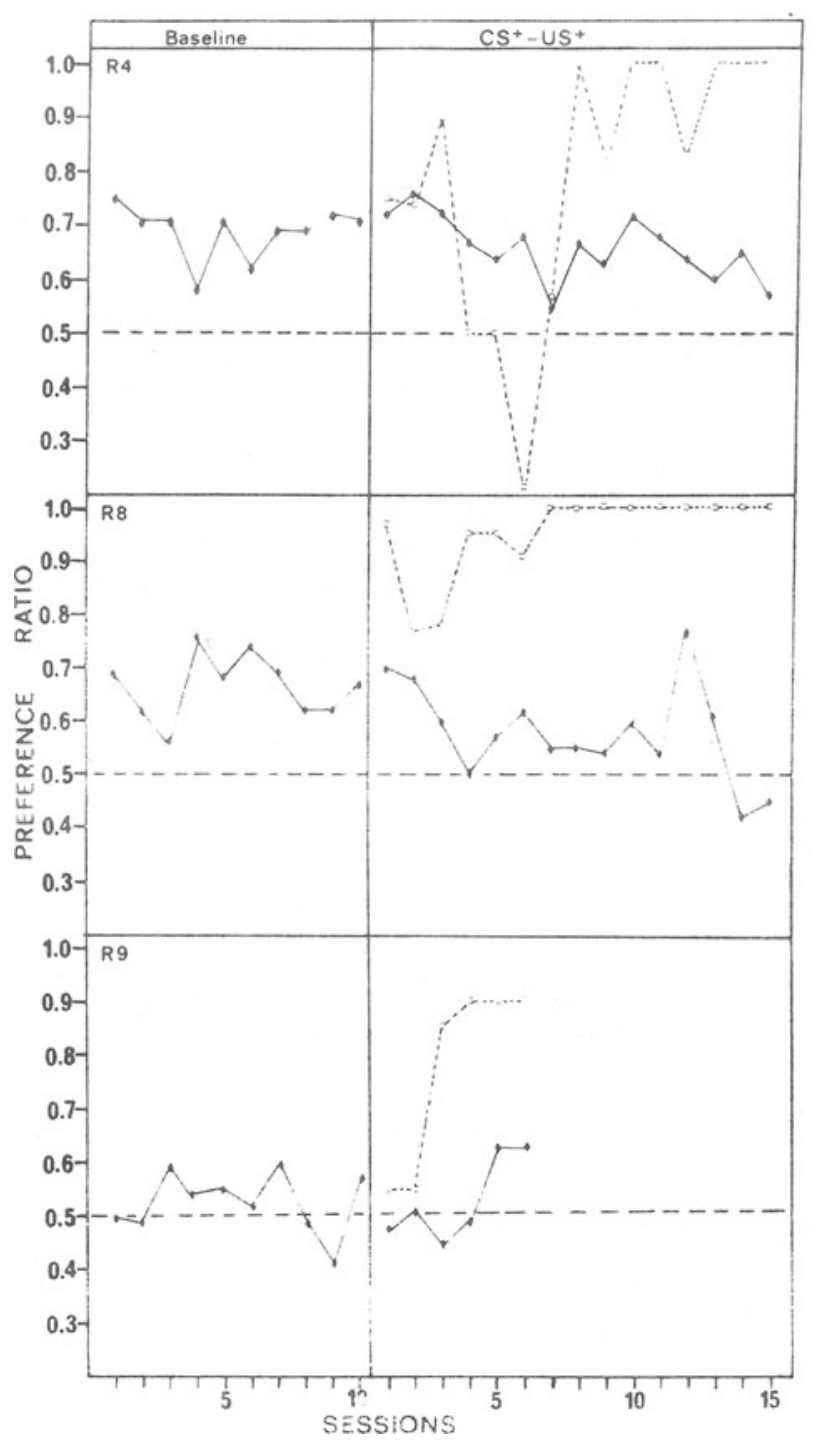

Fig. 1. Preference ratios (relative response rate on the $16 \%$ lever) within (solid lines, squares) and outside (dotted lines, diamonds) CS periods.

After 17 daily 45-min sessions of the above procedure, clicker $\left(\mathrm{CS}^{+}\right)$-pellet $\left(\mathrm{US}^{+}\right)$pairings were introduced every $10 \mathrm{~min}$ on average (range $5-15 \mathrm{~min}$ ) without alteration to the baseline concurrent reinforcement schedule. The CS was $15 \mathrm{sec}$ of clicks and the US was five 45-mg Noyes pellets. These were deposited in a recessed food tray, positioned midway between the two levers. Access to this tray was via a lightly hinged plastic door which, when nosed open, broke a microswitch, which in turn activated a recording circuit. No CS habituation was deemed necessary, as the clicker was also the CS in the previous study (Leslie, 1971). Superimposed pairings were continued for 15 sessions with R4 and R8 and for 6 sessions with R9, who then became ill and could not continue in the experiment.

\section{RESULTS}

The $4 \%-16 \%$ concurrent reinforcement schedule succeeded in producing asymmetric response rates on the two levers. R9 did not invariably show a higher response rate on the $16 \%$ lever, but the $16 \%$ rates were found by a sign test to be significantly greater than the $4 \%$ rates for each $S$ over the last 10 baseline sessions ( $p<.001$ in all cases). The left panel of Fig. 1 shows the preference ratio, calculated as $16 \%$ rate $/ 16 \%$ rate $+4 \%$ rate in each session for each animal over this period. Two animals maintained a preference of about .7, the other (R9) averaged .55. The right panel of Fig. 1 shows the drastic effect of $\mathrm{CS}^{+}-\mathrm{US}^{+}$pairings. Outside the CS, preferences remain relatively undisturbed (solid lines); but within the CS, responding becomes largely confined to the $16 \%$ lever. This is more clearly seen in Fig. 2, which gives the suppression ratio of both responses during the sessions that $\mathrm{CS}^{+}-\mathrm{US}^{+}$pairings were presented. This is calculated as rate within CS/rate outside CS + rate within CS; thus, .05 indicates no effect (below that means suppression and above that means enhancement) during the CS (Kamin, 1961). Here it can be seen that the suppression of $4 \%$ lever responding was common to all Ss. Only R4 showed suppression of $16 \%$ lever responding, and this began to recover after the first six sessions. Otherwise, there was a tendency for $16 \%$ lever responding to be enhanced during CS periods.

\section{DISCUSSION}

The major effect of a CS paired with free food was to suppress selectively the weaker of two concurrent operant responses. In contrast, responding on the stronger operant (the $16 \%$ lever) remained relatively unperturbed. We may immediately reject one possible explanation of this effect. If $\mathrm{Ss}$ were to spend

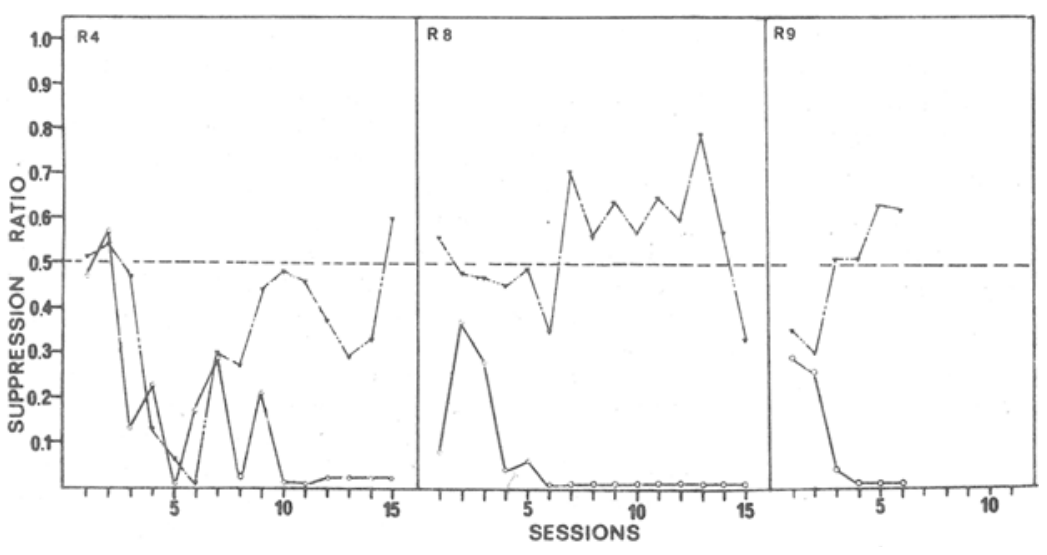

Fig. 2. Suppression ratios for each leverpressing response. Triangles indicate the $16 \%$ lever ratios, circles indicate the $4 \%$ lever ratios. 
considerable time during CS periods making anticipatory food-pellet responses incompatible with leverpressing, then the less probable operant (4\% leverpress) might be maximally disturbed. Anticipatory approaches to the tray in which $\mathrm{US}^{+}$ pellets were dispensed were recorded in and out of the CS and were found to be extremely rare (usually less than one approach per minute), and their frequency did not change appreciably during the CS.

Any temporary disruption of behavior that is maintained by positive reinforcement will produce a drop in obtained reinforcement rate, which in turn may produce response rate reduction (Herrnstein, 1970). Consequently, any significant stimulus superimposed on positively reinforced responding tends to suppress it. However, such generalized suppression cannot account for the shift in preference obtained in this experiment, unless it chanced to produce an increase in relative reinforcement rate of the preferred response, which in turn might have produced the shift in preference. The operation of such a mechanism seems unlikely in this experiment when it is noted that each CS presentation lasted only $15 \mathrm{sec}$ and there were, on average, only four in each session. This meant that an average of one reinforcement would be set up (for each response) during CS presentations in a session, compared with approximately $\mathbf{4 5}$ for each response during the remainder of the session. It seems, then, that virtually total suppression of $4 \%$ lever responding would have been a prerequisite if the obtained relative reinforcement rate were to change; although this occurred late in training for all Ss, it was preceded, for R8 and R9 at least, by the disappearance of any trace of generalized suppression, i.e., the development of a shift in preference, and cannot, therefore, have caused such a shift.

The preference shifts in the present experiment parallel those found by de Villiers \& Millenson (1972). They presented a CS paired with electric shock to rats concurrently responding for 4.5 and $1.5 \mathrm{sec}$ access to diluted condensed milk and found a similar increase in preference for the response producing the larger reinforcements. de Villiers and Millenson interpreted this preference shift as support for the view that a CS paired with an aversive event subtracts equally from the two incentive values sustaining the two positively reinforced operants. Thus, if $A$ is the assumed reinforcing value of responding for $4.5 \mathrm{sec}$ access, B is that for $1.5 \mathrm{sec}$ access, and $\mathrm{C}$ is the decrement produced by the $\mathrm{CS}$, then as $\mathrm{A}-\mathrm{C} / \mathrm{B}-\mathrm{C}>\mathrm{A} / \mathrm{B}$ (with $\mathrm{A}>\mathrm{B}>\mathrm{C}>0$ ), preference increases during the $\mathrm{CS}$.

The similar shift found in the present experiment suggests that the effect of a CS (formally the sign of C) superimposed on positively reinforced response rates is independent of the sign of the US used in the pairing. This formulation does not explain Estes's $(1943,1948)$ finding that the presentation of a CS that had previously been paired with food enhanced the rate of a positively reinforced operant during extinction; however, it accords with Azrin \& Hake's (1969) suggestion that pairing a CS with any strong reinforcer, positive or negative, produces a general emotional state. Such a state could reduce the values of the reinforcement, sustaining ongoing responses directly (Estes, 1969), or it could do so indirectly by raising the probability of incompatible competing responses (operants or respondents). Suppose that in a two-response asymmetrically reinforced concurrent schedule the stronger operant is less susceptible to such disruptions, regardless of whether they are produced by positively or negatively signed emotional states. Then similar preference shifts would be expected in both this procedure and that used by de Villiers \& Millenson (1972).

Despite this common result, we do not wish to deny the data of casual observation and experiment which concur in distinguishing many important behavioral effects induced by conditioned stimuli associated with reinforcers of different sign. For instance, Kelly (1971) presented two functionally different CSs in the same session to monkeys that were leverpressing for food pellets. One CS preceded shock, the other preceded "free food." Both stimuli affected leverpressing similarly, suppressing it increasingly throughout the 3-min CS period. However, their effects on two cardiovascular responses were qualitatively different. Unsystematic observations of Ss in this and the previous study (Leslie, 1971) showed patterns of behavior which, although varied, in no way resembled the freezing, defecation, and urination seen when the CS is paired with shock. Thus, while some features of emotional states may be general, e.g., their ability to interrupt behavior sequences, others remain peculiar to the particular state.

The present data suggest the following conclusion: One effect of a CS paired with food or shock on positively reinforced response rates is to modify the organism's hierarchy of reinforcement values in such a way as to lower temporarily the relative value of the baseline reinforcer by temporarily strengthening the value of some competing reinforcer or reinforcers. In a single operant situation, this change is seen as suppression of the reference response. In a two-response concurrent, it is seen as a relatively larger suppression of the weaker of the two reference operants, hence, a preference shift. Evidently the adequacy of this account depends upon identification of those competing reinforcers.

\section{REFERENCES}

Azrin, N. H., \&z Hake, D. F. Positive conditioned suppression: Conditioned suppression using positive reinforcers as the unconditioned stimuli. Journal of the Experimental Analysis of Behavior, $1969,12,167-173$

Bindra, D., \& Palfai, T. Nature of positive and negative incentive-motivational effects on general activity. Journal of Comparative \& Physiological Psychology, 1967, 63, 288-297.

Bryant, R. C. Conditioned suppression of free-operant avoidance. Journal of the Experimental Analysis of Behavior 1972, 17, 257-260.

Catania, A. C. Concurrent operants. In W. K. Honig (Ed.), Operant behavior: Areas of research and application. New York: Appleton-Century-Crofts, 1966. Pp. 213-270.

de Villiers, P. A., \& Millenson, J. R. Concurrent performances: A baseline for the study of conditioned anxiety. Journal of the Experimental Analysis of Behavior, 1972.

Estes, W. K. Discriminative conditioning: I. A discriminative property of conditioned anticipation. Journal of Experimental Psychology, 1943, 32, 150-155.

Estes, W. K. Discriminative conditioning: II. Effects of a Pavlovian conditioned stimulus upon a subsequently acquired operant response. Journal of Experimental Psychology, 1948, 38, 173-177.

Estes, W. K. Outline of a theory of punishment. In B. A. Campbell and R. M. Church (Eds.), Punishment and aversive behavior. New York: Appleton-Century-Crofts, 1969. Pp. 57-82.

Estes, W. K., \& Skinner, B. F. Some quantitative properties of anxiety. Journal of Experimental Psychology, 1941, 29, 390-400.

Hammond, L. J. Conditioned emotional states. In P. Black (Ed.), Physiological correlates of emotion. New York: Academic Press, 1970. Pp. 245-259.

Herrnstein, R. J. On the law of effect. Journal of the Experimental Analysis of Behavior, 1970, 13, 243-266.

Kamin, L. J. Trace conditioning of the conditioned emotional response. Journal of Comparative \& Physiological Psychology, $1961,54,149-153$.

Kelly, D. D. The contrasting cardiovascular consequences of monkeying around with conditioned anxiety, conditioned elation, and discriminated extinction. Paper presented at the annual meeting of the Eastern Psychological Association, New York, April 1971.

Leslie, J. C. The effects of a stimulus paired with food reinforcement on the behavior of rats barpressing for sucrose. Unpublished BA Thesis, University of Oxford, 1971.

LoLordo, V. M. Facilitation of food-reinforced responding by a signal for response-independent food. Journal of the Experimental Analysis of Behavior, 1971, 15, 49-55.

Meltzer, D., \& Brahlek, J. A. Conditioned suppression and conditioned enhancement with the same positive UCS: An effect CS duration. Journal of the Experimental Analysis of Behavior, 1970, 13, 67-70.

Millenson, J. R. Principles of behavioral analysis. New York: Macmillan, 1967

Millenson, J. R. A programming language for on-line control of psychological experiments. Behavioral Science, 1971, 16, 248-256.

Millenson, J. R., \& de Villiers, P. A. Motivational properties of conditioned anxiety. In $R$. M. Gilbert and J. R. Millenson (Eds.), Reinforcement: Behavioral analyses. New York: 
Academic Press, 1972.

Mizcek, K. A., \& Grossman, S. P. Positive conditioned suppression: Effects of CS duration. Journal of the Experimental Analysis of Behavior, 1971, 15, 243-247.

Mowrer, O. H. Learning theory and behavior. New York: Wiley, 1960.

Rescorla, R. A. Inhibition of delay in Pavlovian fear conditioning. Journal of Comparative \& Physiological Psychology, 1967, 64, 114-120.

Rescorla, R. A., \& Solomon, R. L. Two process learning theory: Relationship between Pavlovian conditioning and instrumental learning. Psychological Review, 1967, 74, 151-182.

Scobie, S. R. Interaction of an aversive Pavlovian conditional stimulus with aversively and appetitively motivated operants in rats. Journal of Comparative \& Physiological Psychology, 1972, 79, 171-188.
Shull, R. L., \& Pliskoff, S. S. Changeover delay and concurrent performances: Some effects on relative performance measures. Journal of the Experimental Analysis of Behavior, 1967, 10, 517-527.

Stubbs, D. A., \& Plisk off, S. S. Concurrent responding with fixed relative rate of reinforcement. Journal of the Experimental Analysis of Behavior, 1969, 12, 887-895.

\section{NOTE}

1. Throughout the notation $\mathrm{CS}^{+}-\mathrm{US}^{+}$refers to a conditioned stimulus (CS) positively correlated $(+)$ with an unconditioned stimulus (US) which is also a positive $(+)$ reinforcer.

(Received for publication November 6, 1972.) 